\title{
Acurácia e confiabilidade na medida da glicemia em pacientes críticos adultos: revisão integrativa
}

\author{
Laura Menezes Silveira ${ }^{1}$ \\ Simone Costa Silva ${ }^{2}$ \\ Maíza Cláudia Vilela Hipólito ${ }^{3}$ \\ Simone de Godoy 4 \\ Angelita Maria Stabile 5
}

\section{RESUMO}

A mensuração fidedigna da glicemia no paciente crítico é essencial para a assistência segura. Vieses na medição podem resultar na obtenção de valores imprecisos, levando a equipe a interpretar equivocadamente os resultados, modificando erroneamente as condutas terapêuticas. Neste estudo, objetivou-se buscar na literatura a produção existente sobre os fatores relacionados a acurácia e a confiabilidade das medidas de glicemia de pacientes assistidos em UTI, por meio de busca nas bases de dados Cinahl, Pubmed, Web of Science, Scopus e Biblioteca Virtual em Saúde. As evidências de 18 artigos que versavam sobre a acurácia e a confiabilidade da verificação da glicemia, mostraram aspectos como: método de mensuração, tipo de amostra, características do paciente crítico e manuseio do aparelho. A equipe deve ponderar sobre as vantagens e limitações de cada método considerando a sua realidade e a segurança do paciente.

Descritores: Glicemia; Confiabilidade dos Dados; Cuidados Críticos; Unidades de Terapia Intensiva; Testes Imediatos.

\footnotetext{
${ }^{1}$ Enfermeira, Mestre em Enfermagem Fundamental. Discente do Programa de Pós-Graduação em Enfermagem Fundamental, nível Doutorado, da Escola de Enfermagem de Ribeirão Preto da Universidade de São Paulo. Ribeirão Preto, SP, Brasil. E-mail: lauramsilveira@yahoo.com.br.

${ }^{2}$ Enfermeira. Discente do Programa de Pós-Graduação em Enfermagem Fundamental, nível Mestre, da Escola de Enfermagem de Ribeirão Preto da Universidade de São Paulo. Ribeirão Preto, SP, Brasil. E-mail: si.costa@yahoo.com.

${ }^{3}$ Enfermeira, Mestre em Educação Física. Discente do Programa de Pós-Graduação em Educação Física, nível Doutorado, Faculdade de Educação Física da Universidade Estadual de Campinas. Campinas, SP, Brasil. E-mail: maizavilela@yahoo.com.br.

${ }^{4}$ Enfermeira, Doutora em Enfermagem. Professor Doutor da Escola de Enfermagem de Ribeirão Preto da Universidade de São Paulo. Ribeirão Preto, SP, Brasil. E-mail: sig@eerp.usp.br.

${ }^{5}$ Enfermeira, Doutora em Fisiologia. Professor Doutor da Escola de Enfermagem de Ribeirão Preto da Universidade de São Paulo. Ribeirão Preto, SP, Brasil. E-mail: angelita@eerp.usp.br.
}

\section{Como citar esse artigo:}

Silveira LM, Silva SC, Hipólito MCV, Godoy S, Stabile AM. Acurácia e confiabilidade na medida da glicemia em pacientes críticos adultos: revisão integrativa. Rev. Eletr. Enf. [Internet]. 2018 [acesso em: ];20:v20a03. Disponível em: https://doi.org/10.5216/ree.v20.46567. 


\section{INTRODUÇÃO}

Alterações nos níveis glicêmicos são comuns em pacientes críticos. A reação inflamatória, o estresse ocasionado pela resposta do organismo à doença e os tratamentos implementados podem ocasionar resistência à insulina, intolerância à glicose e hiperglicemia ${ }^{(1-2)}$. A hiperglicemia é considerada um marcador de gravidade, fortemente associado ao aumento do risco de morte do paciente ${ }^{(3-5)}$, pois gera desequilíbrio do sistema imunológico e da resposta inflamatória que se torna inespecífica, resultando em estresse oxidativo, disfunção mitocondrial, morte celular e injúria tecidual com consequente falência de órgãos ${ }^{(6-9)}$.

Diante desse cenário, identifica-se na literatura estudos realizados em serviços de cuidados intensivos que buscaram a manutenção dos valores glicêmicos com o mínimo de variabilidade, o que reduziu significativamente os índices de morbimortalidade ${ }^{(3,10-14)}$. Salienta-se que, para regularizar a glicemia são empregadas intervenções como a administração de insulina em infusão contínua que pode induzir ao risco de hipoglicemia a qual pode ser mais nociva do que a hiperglicemia(3,10-15).

Nesse contexto, o monitoramento glicêmico na Unidade de Terapia Intensiva (UTI) é um cuidado rotineiro ${ }^{(5)}$ viabilizado por meio de mensurações da glicemia a fim de ajustar a administração de insulina ou de glicose ${ }^{(13,16)}$.

Atualmente, para o controle glicêmico nas Unidades de Terapia Intensiva (UTI), são utilizados dispositivos de monitoramento contínuo (sensores subcutâneos ou intravenosos)(17-18) e intermitentes (glicosímetros, hemogasômetros e laboratoriais)(16,19-20), sendo o glicosímetro portátil bastante utilizado para verificação da glicemia, devido a sua praticidade, baixo custo e fácil manuseio ${ }^{(21)}$. Além de ser um método prático, a mensuração da glicemia utilizando este dispositivo e fitas apropriadas, possibilita a utilização de amostras de sangue arterial, venoso ou capilar ${ }^{(21-22)}$, podendo ser realizado com frequência, a depender da necessidade clínica do paciente e dos níveis obtidos nas verificações, permitindo intervenções terapêuticas imediatas e reduzindo complicações decorrentes do descontrole metabólico.

A mensuração acurada e confiável da glicemia pode ser determinante para o desfecho do quadro do paciente e para a assistência segura. Acredita-se que a obtenção de valores imprecisos e/ou não confiáveis levam a equipe a interpretar equivocadamente os resultados e modificar as condutas terapêuticas ${ }^{(22-23)}$. Medidas precisas orientam as prescrições de insulina ou glicose e auxiliam na terapia nutricional ${ }^{(24-25)}$ a fim de facilitar a recuperação do estresse fisiológico que ocorre durante tratamento crítico.

Nessa perspectiva, considera-se que reduzir as oscilações dos níveis glicêmicos e determinar uma faixa de variação glicêmica segura para o paciente crítico, é tão importante quanto obter valores de glicemia confiáveis. Sendo assim, o objetivo deste estudo foi buscar na literatura científica a produção existente sobre os fatores relacionados a acurácia e a confiabilidade da mensuração da glicemia no paciente crítico assistido em UTI.

\section{MÉTODO}

Trata-se de uma revisão integrativa da literatura com a finalidade de reunir e sintetizar as evidências disponíveis em artigos originais produzidos sobre o tema. O estudo foi composto por seis etapas, as quais compreenderam: definição do problema e objetivo da pesquisa (identificação do tema); estabelecimento de critérios de inclusão e exclusão das publicações e busca na literatura; categorização dos estudos primários 
(definição das informações a serem extraídas dos estudos selecionados); análise dos estudos incluídos na revisão integrativa; interpretação dos resultados; e síntese do conhecimento disseminado pelas publicações ${ }^{(26-27)}$.

Para a elaboração da questão norteadora, utilizou-se a estratégia "PICO" que é representada por um acrônimo para Paciente, Intervenção, Comparação e "Outcomes" (desfecho) a fim de localizar as melhores evidências disponíveis, na busca em cada base de dados ${ }^{(28)}$. Nesse estudo, o acrônimo PICO foi utilizado da seguinte maneira: P - Pacientes críticos assistidos em unidade terapia intensiva, I - Mensuração da glicemia, Cnão se aplica, e O- acurácia e confiabilidade.

A questão norteadora foi definida como: "Quais as evidências disponiveis na literatura sobre os fatores relacionados à acurácia e a confiabilidade da mensuração da glicemia no paciente crítico assistido em UTI?". Para o levantamento dos artigos, foram utilizadas as bases de dados Pubmed, Web of Science, CINAHL (Cumulative Index to Nursing and Allied Health Literature) e Scopus e a biblioteca BVS (Biblioteca Virtual de Saúde). As buscas dos artigos foram realizadas nas bases de dados e biblioteca, utilizando os descritores controlados "Critical Care" (cuidados críticos), "Point-of-care testing" (teste imediato), "Blood Glucose" (glicemia) e "Data Accuracy" (confiabilidade dos dados). Os descritores foram definidos com auxílio do DeCs (Descritores em Ciências da Saúde) ou Mesh (Medical Subject Headings) utilizados em conjunto com o operador booleano "AND". Ressalta-se que foram realizados testes nas bases de dados e biblioteca utilizando palavras chave, como "reliability" (confiabilidade) e "feasibility" (viabilidade), combinadas com os descritores acima, entretanto, os estudos revelados nessa estratégia de busca já haviam sido localizados nas buscas anteriores.

Foram considerados elegíveis os artigos primários, publicados na íntegra, que versavam sobre a acurácia e confiabilidade da verificação da glicemia no paciente crítico adulto assistido em UTI, publicados nos idiomas inglês, espanhol e português, no período determinado entre janeiro de 2006 a dezembro de 2016, a fim de analisar evidências mais recentes sobre o tema. Não foram incluídos estudos cujas amostras foram constituídas exclusivamente por pacientes neonatos, pediátricos, diabéticos, queimados, cardiocirúrgicos e neurocirúrgicos devido às especificidades dessas condições.

Para a extração dos dados dos artigos incluídos, foi adaptado um instrumento validado, que engloba a identificação do artigo, os autores, o tipo de publicação, o detalhamento metodológico, o detalhamento amostral, a intervenção estudada, os resultados e as recomendações/conclusões ${ }^{(29)}$.

Para compreensão dos resultados, destaca-se os termos acurácia como a capacidade de determinar o valor verdadeiro, expressa pela diferença entre a média de muitos valores de medidos e o valor "verdadeiro" ou a concentração conhecida de glicose (método considerado referência) ${ }^{(30-31)}$, confiabilidade é a capacidade de um teste reproduzir o mesmo resultado quando repetido e/ou de métodos que possuem a mesma finalidade obterem os mesmos resultados, expresso pela distribuição de medidas individuais em torno de um valor médio( ${ }^{(30)}$. As análises de correlação e regressão, Bland-Altman e as grades de erro Clarke são algumas das métricas utilizadas para demonstrar acurácia e confiabilidade nos estudos ${ }^{(21)}$.

As publicações selecionadas foram classificadas de acordo com o delineamento metodológico e o nível de evidência, e a síntese dos dados está apresentada de forma descritiva.

Os níveis de evidência foram caracterizados de forma hierárquica de acordo com a abordagem metodológica adotada: nível I de evidência a revisão sistemática ou meta-análise de ensaios clínicos randomizados 
controlados (ECRC) relevantes, ou de diretrizes clínicas, baseadas em revisões sistemáticas de ECRC; é designado nível II a evidência derivada de um ou mais ECRC adequadamente delineados; qualifica-se como nível III a evidência obtida de ensaios clínicos devidamente delineados, sem randomização; o nível IV é constituído pela evidência proveniente de estudo de caso-controle ou coorte com delineamento apropriado; rotula-se como nível V a evidência proveniente de revisão sistemática de estudos qualitativos e descritivos; o nível VI é composto por evidência derivada de um único estudo descritivo ou qualitativo; a evidência nível VII é aquela oriunda da opinião de autoridades e/ou relatórios de comitês de especialistas ${ }^{(32)}$.

Figura 1: Processo de seleção de artigos incluídos na revisão integrativa. Ribeirão Preto, (SP), 2017.

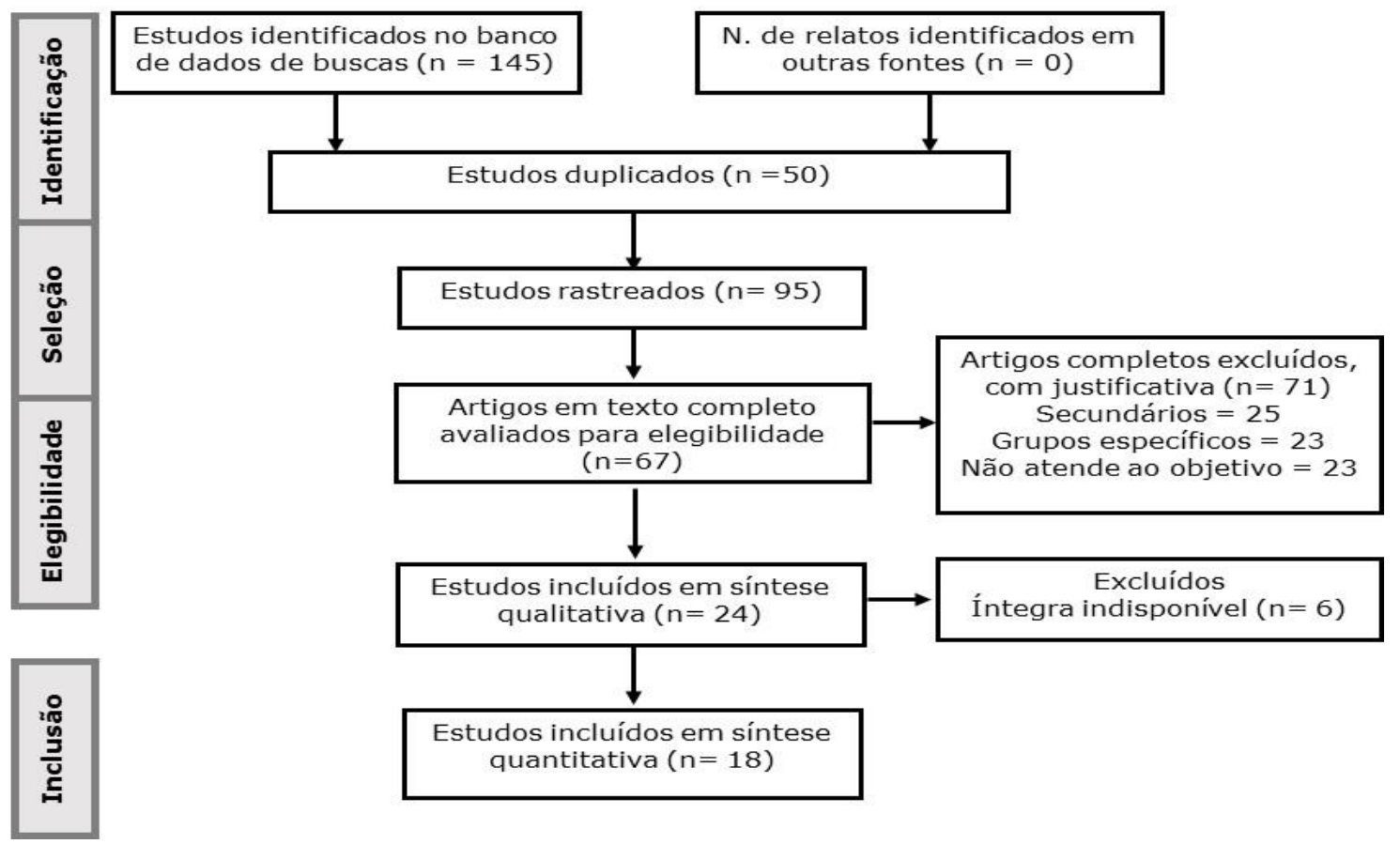

Fonte: Fluxograma elaborado pelos autores conforme as recomendações de Moher et al. $(2015)^{(33)}$.

\section{RESULTADOS}

A amostra final foi composta por 18 artigos. A análise dos artigos incluídos nesta revisão evidenciou dados sobre confiabilidade e acurácia das medidas de glicemia considerando amostras que incluíram pacientes críticos. O Quadro 1 apresenta as características dos artigos selecionados para esta revisão. 
Quadro 1: Artigos incluídos na revisão integrativa conforme autor, ano de publicação, nível de evidência e país de origem, amostra, delineamento e método e principais resultados.

\begin{tabular}{|c|c|c|c|}
\hline $\begin{array}{l}\text { Autor, ano, País de procedência, } \\
\text { Nível de evidência }\end{array}$ & Amostra, Idade & $\begin{array}{l}\text { Delineamento do estudo, métodos de mensuração } \\
\text { da glicemia }\end{array}$ & Principais Resultados \\
\hline Pereira et al., $2015^{(34)}$, Brasil, IV. & $\begin{array}{l}145 \text { pacientes, média de idade de } \\
660,9 \text { anos. }\end{array}$ & $\begin{array}{c}\text { Prospectivo, transversal. } \\
\text { Dispositivos utilizados: Precision PCx (Abbott, Illinois, } \\
\text { USA) e Accu-chek Advantage II glucometer (Roche, } \\
\text { Basel, Switzerland). } \\
\text { Método referência: laboratorial. } \\
\text { Tipo de amostra de sangue: arterial (cateter } \\
\text { permanente), capilar e venoso (cateter central). }\end{array}$ & $\begin{array}{c}\text { Acurácia e Confiabilidade: } \\
\text { Os dois glicosímetros testados apresentaram baixa } \\
\text { acurácia e imprecisão, principalmente quando utilizou-se } \\
\text { sangue venoso do CVC. Houve variação entre os resultados } \\
\text { das medidas entre os métodos. } \\
\text { Fatores relacionados ao paciente: } \\
\text { Redução do nível de hematócrito e acidose foram } \\
\text { associados com diferenças entre as medidas de glicemia } \\
\text { com sangue arterial e venoso. O uso vasopressores } \\
\text { aumentou o erro nas medidas com sangue capilar. }\end{array}$ \\
\hline $\begin{array}{l}\text { Van Hooijdonk et al., 2015(35), } \\
\text { Holanda, IV. }\end{array}$ & $\begin{array}{c}50 \text { pacientes, } 105 \text { sensores, } 929 \\
\text { amostras, média de idade } 65 \text { anos. }\end{array}$ & $\begin{array}{c}\text { Prospectivo. } \\
\text { Dispositivo utilizados: Monitor Subcutâneo contínuo } \\
\text { de glicemia Medtronic MiniMed (Medtronic Inc., } \\
\text { Northridge, CA, EUA). } \\
\text { Método de referência: analisador de gases RapidLab } \\
1265 \text { (Siemens Healthcare Diagnostics, The Hague, The } \\
\text { Netherlands). } \\
\text { Tipo de amostra de sangue: arterial. }\end{array}$ & $\begin{array}{c}\text { Acurácia e Confiabilidade: } \\
\text { O monitor subcutâneo contínuo não cumpriu as normas } \\
\text { ISO 15197:2003. } \\
\text { Fatores relacionados ao paciente: } \\
\text { O dispositivo foi mais preciso em pacientes diabéticos. } \\
\text { Fatores relacionados ao manuseio do aparelho: } \\
\text { O aumento do número de calibrações melhorou a precisão } \\
\text { do medidor contínuo de glicemia. }\end{array}$ \\
\hline $\begin{array}{c}\text { Garingarao et al., 2014(36), Filipinas, } \\
\text { IV. }\end{array}$ & $\begin{array}{l}180 \text { pacientes sendo } 89 \\
\text { normotensos - média de idade 51,3 } \\
\text { anos e } 91 \text { hipotensos - média de } \\
\text { idade } 54,7 \text { anos, } 186 \text { medidas. }\end{array}$ & $\begin{array}{c}\text { Prospectivo, transversal. } \\
\text { Dispositivo utilizado: glicosímetro Accu-Chek Active } \\
\text { Meter (Roche Diagnostics, Indianapolis, Indiana, USA). } \\
\text { Método de referência: laboratorial. } \\
\text { Tipo de amostra de sangue: venoso de veia periférica } \\
\text { ou de cateter central e capilar. }\end{array}$ & $\begin{array}{l}\text { Acurácia e Confiabilidade: } \\
\text { No grupo normotenso as medidas do glicosímetro foram } \\
\text { concordantes. No grupo hipotenso a concordância foi de } \\
79 \% \text { descumprindo a recomendação das } \\
\text { ISO 15197:2003. } \\
\text { Fatores relacionados ao paciente: } \\
\text { Frequência cardíaca e respiratória elevadas, menor } \\
\text { pontuação na escala de Glasgow, necessidade de } \\
\text { ventilação mecânica com acidose lática, redução de } \\
\text { leucócitos podem estar associados ao menor desempenho } \\
\text { do glicosímetro no grupo hipotenso. }\end{array}$ \\
\hline
\end{tabular}




\begin{tabular}{|c|c|c|c|}
\hline $\begin{array}{l}\text { Autor, ano, País de procedência, } \\
\text { Nível de evidência }\end{array}$ & Amostra, Idade & $\begin{array}{l}\text { Delineamento do estudo, métodos de mensuração } \\
\text { da glicemia }\end{array}$ & Principais Resultados \\
\hline Lonjaret et al., 2012(37), França, IV. & $\begin{array}{l}75 \text { pacientes, } 304 \text { medidas, média } \\
\text { de idade } 59 \text { anos. }\end{array}$ & $\begin{array}{c}\text { Prospectivo, observacional. } \\
\text { Dispositivo utilizado: } \\
\text { glicosímetro CONTOUR }{ }^{\circledR} \text { TS, Bayer HealthCare, } \\
\text { Tarrytown, NY, USA) } \\
\text { Método de Referência: laboratorial. } \\
\text { Tipo de amostra de sangue: capilar e arterial }\end{array}$ & $\begin{array}{l}\text { Acurácia e Confiabilidade } \\
\text { As amostras arteriais foram mais precisas do que as } \\
\text { amostras capilares. } \\
\text { Os resultados do glicosímetro não atenderam aos critérios } \\
\text { ISO 15971:2003. } \\
\text { Os resultados das medidas utilizando sangue capilar foram } \\
\text { subestimados. } \\
\text { Fatores relacionados ao paciente: } \\
\text { Uso de insulina, nível de lactato, uso de drogas vasoativas } \\
\text { reduz a precisão da medida independente do tipo de } \\
\text { amostra de sangue. }\end{array}$ \\
\hline Juneja et al., 2011(38), Índia, IV & $\begin{array}{l}200 \text { pacientes, } \\
\text { sendo o grupo de estudo } 100 \text { com } \\
\text { choque séptico, hipotensos em uso } \\
\text { de vasopressor - média de idade } 63 \\
\text { anos e grupo controle } 100 \\
\text { pacientes normotensos - média de } \\
\text { idade } 63,9 \text { anos. }\end{array}$ & $\begin{array}{l}\text { Prospectivo, caso controle. } \\
\text { Dispositivo utilizado: One Touch Ultra Blood Glucose } \\
\text { Monitoring System (LifeScan, Johnson \& Johnson). } \\
\text { Método de referência: não mencionado. } \\
\text { Tipo de amostra de sangue: capilar e arterial. }\end{array}$ & $\begin{array}{c}\text { Acurácia e confiabilidade: } \\
\text { Os resultados das medidas utilizando sangue arterial e } \\
\text { capilar não foram confiáveis no grupo hipotenso. A } \\
\text { precisão do medidor não estava de acordo os critérios ISO } \\
\text { 15197:2003. } \\
\text { No grupo controle os resultados foram confiáveis. } \\
\text { Fatores relacionados ao paciente: } \\
\text { Hipotensão e uso de vasopressor reduzem a precisão do } \\
\text { medidor utilizado. }\end{array}$ \\
\hline $\begin{array}{l}\text { Fekih Hassen et al. 2010(39); Tunísia, } \\
\text { IV. }\end{array}$ & $\begin{array}{l}43 \text { pacientes hiperglicêmicos sendo } \\
\text { grupo } 1 \text { - } 23 \text { pacientes } \\
\text { hemodinamicamente estáveis - } \\
\text { média de idade } 57 \text { anos; e grupo } 2 \text { - } \\
20 \text { pacientes em uso de } \\
\text { catecolaminas, média de idade } 64 \\
\text { anos. }\end{array}$ & $\begin{array}{c}\text { Prospectivo. } \\
\text { Dispositivo utilizado: Accu-Chek (Roche Diagnostics, } \\
\text { Mannheim, Germany). } \\
\text { Método de referência: laboratorial. } \\
\text { Tipo de amostra de sangue: capilar sendo uma da } \\
\text { polpa digital e outra da orelha) e venoso. }\end{array}$ & $\begin{array}{c}\text { Acurácia e confiabilidade: } \\
\text { As medidas realizadas com o glicosímetro não foram } \\
\text { precisas no grupo } 2 \text { (suporte vasopressor), independente } \\
\text { do sítio de coleta (orelha ou polpa digital). A coleta de } \\
\text { sangue na orelha não melhorou a precisão do resultado da } \\
\text { glicemia. } \\
\text { Fatores relacionados ao paciente: } \\
\text { Uso de catecolaminas reduz a acurácia do glicosímetro. }\end{array}$ \\
\hline $\begin{array}{l}\text { Karon et al. 2009(40), Estados Unidos } \\
\text { da América (EUA), IV. }\end{array}$ & 50 pacientes, idade não informada. & $\begin{array}{c}\text { Prospectivo. } \\
\text { Dispositivos utilizados: Accu-Chek Inform (Roche } \\
\text { Diagnostics, Indianapolis, IN) e Stat-Strip (Nova } \\
\text { Biomedical, Waltham, MA), } \\
\text { Método de referência: laboratorial. } \\
\text { Tipo de amostra de sangue: venoso provenientes de } \\
\text { CVC ou PICC, venoso por punção periférica. }\end{array}$ & $\begin{array}{l}\text { Acurácia e confiabilidade: } \\
\text { O glicosímetro Accu-Chek Inform mostrou diferenças de } \\
\text { até } 20 \mathrm{mg} / \mathrm{dL} \text { entre as suas medidas e o laboratório. O Stat- } \\
\text { Strip apresentou valores superestimados, principalmente } \\
\text { nas amostra de sangue do CVC. }\end{array}$ \\
\hline
\end{tabular}




\begin{tabular}{|c|c|c|c|}
\hline $\begin{array}{l}\text { Autor, ano, País de procedência, } \\
\text { Nível de evidência }\end{array}$ & Amostra, Idade & $\begin{array}{l}\text { Delineamento do estudo, métodos de mensuração } \\
\qquad \text { da glicemia }\end{array}$ & Principais Resultados \\
\hline $\begin{array}{l}\text { Hoedemarekers et al. } 2008^{(41)} \text {, EUA, } \\
\text { IV. }\end{array}$ & $\begin{array}{c}\text { Três grupos: } \\
-85 \text { pacientes críticos, (197 } \\
\text { amostras) } \\
\text { - } 53 \text { pacientes críticos ( } 82 \text { amostras) } \\
-47 \text { pacientes não críticos ( } 74 \\
\text { amostras) } \\
\text { Média de idade } 55 \text { anos. }\end{array}$ & $\begin{array}{c}\text { Prospectivo, observacional. } \\
\text { Dispositivos utilizados: Accu-Chek Sensor (Roche } \\
\text { Diagnostics, Abbott Park, IL), Precision (Abbott } \\
\text { Diagnostics) e HemoCue, Sweden. } \\
\text { Método de referência: laboratorial. } \\
\text { Tipo de amostra de sangue: arterial. }\end{array}$ & $\begin{array}{l}\text { Acurácia e confiabilidade: } \\
\text { Os resultados de glicemia dos três dispositivos não } \\
\text { atenderam aos critérios ISO. } \\
\text { Fatores relacionados ao paciente: } \\
\text { Pacientes com pontuação maior nos escores de gravidade } \\
\text { e prognóstico tiveram medidas mais imprecisas, que foram } \\
\text { superestimados, induzindo a administração desnecessária } \\
\text { de insulina. }\end{array}$ \\
\hline Price et al., 2008(42), Reino Unido, IV. & $\begin{array}{l}17 \text { pacientes adultos, } 1.101 \\
\text { medições, divididas conforme } \\
\text { comparações: } \\
\text { monitor continuo de glicose com o } \\
\text { glicosímetro e glicosímetro com } \\
\text { tipo de amostras de sangue } \\
\text { diferentes. A média de idade variou } \\
\text { de acordo os grupos de diagnóstico. }\end{array}$ & $\begin{array}{c}\text { Retrospectivo. } \\
\text { Dispositivos utilizados: Monitor continuo de glicemia } \\
\text { “Guardian" (CGMS Gold, Medtronic MiniMed, } \\
\text { Northridge Calif, USA) e o glicosímetro Accu-Chek } \\
\text { Advantage, F Hoffman-La Roche, Basel, Switzerland). } \\
\text { Método de Referência: glicosímetro Accu-Chek } \\
\text { Advantage. } \\
\text { Tipo de amostra de sangue: capilar e arterial. }\end{array}$ & $\begin{array}{l}\text { Acurácia e confiabilidade: } \\
\text { As medidas do monitor foram mais similares às do } \\
\text { glicosímetro utilizando sangue arterial, mas o monitor } \\
\text { superestimou os valores. } \\
\text { Os resultados do glicosímetro não foram confiáveis } \\
\text { independente do tipo de amostra de sangue. }\end{array}$ \\
\hline Critchell et al. $2007^{(43)}$, EUA, IV. & $\begin{array}{l}80 \text { pacientes, média de idade 58,8 } \\
\text { anos, } 277 \text { amostras. }\end{array}$ & $\begin{array}{l}\text { Prospectivo, observacional. Dispositivo utilizado: } \\
\text { glicosímetro Accu-Check Inform (Roche Diagnostics, } \\
\text { Mannheim, Germany). } \\
\text { Método de referência: } \\
\text { laboratorial. } \\
\text { Tipo de amostra de sangue: capilar e venoso. }\end{array}$ & $\begin{array}{c}\text { Acurácia e confiabilidade: } \\
\text { Os resultados das medidas do glicosímetro foram } \\
\text { superestimadas e 19\% não estavam de acordo com os } \\
\text { critérios ISO. O glicosímetro foi mais preciso utilizando } \\
\text { sangue capilar. } \\
\text { Fatores relacionados ao paciente: } \\
\text { O nível de hematócrito, edema e o uso de vasopressores } \\
\text { podem interferir na precisão da medida capilar em } \\
\text { pacientes críticos que apresentavam estas alterações. }\end{array}$ \\
\hline Lacara, et al. $2007^{(44)}$, EUA, IV. & $\begin{array}{l}49 \text { pacientes, média de idade } 66,8 \\
\text { anos. }\end{array}$ & $\begin{array}{c}\text { Prospetivo. } \\
\text { Dispositivo utilizado: } \\
\text { SureStep Pro Hospital Meter e SureStep Pro Hospital } \\
\text { Products Test Strips, Johnson \& Johnson, Milpitas, } \\
\text { California). } \\
\text { Método de referência: laboratorial. } \\
\text { Tipo de amostra de sangue: arterial ou do CVP, capilar } \\
\text { para o teste com glicosímetro }\end{array}$ & $\begin{array}{l}\text { Acurácia e confiabilidade: } \\
\text { A acurácia e confiabilidade do glicosímetro foi maior } \\
\text { utilizando sangue capilar do que amostras de sangue de } \\
\text { cateteres arteriais e CVP. } \\
\text { Fatores relacionados ao paciente: } \\
\text { Níveis inferiores de hematócrito, dióxido de carbono } \\
\text { inferiores reduziram a confiabilidade do glicosímetro. O } \\
\text { mesmo aparelho apresentou falsa elevação dos valores } \\
\text { utilizando sangue arterial. A pressão arterial média não } \\
\text { influenciou os resultados do glicosímetro. }\end{array}$ \\
\hline
\end{tabular}




\begin{tabular}{|c|c|c|c|}
\hline $\begin{array}{l}\text { Autor, ano, País de procedência, } \\
\text { Nível de evidência }\end{array}$ & Amostra, Idade & $\begin{array}{l}\text { Delineamento do estudo, métodos de mensuração } \\
\text { da glicemia }\end{array}$ & Principais Resultados \\
\hline Corstjens et al., 2006 ${ }^{(45)}$, EUA, IV. & $\begin{array}{l}45 \text { pacientes, a idade variou entre } \\
\qquad 31 \text { a } 88 \text { anos. }\end{array}$ & $\begin{array}{l}\text { Prospectivo, observacional. } \\
\text { Dispositivos utilizados: analisador de gases (ABL715 } \\
\text { Series (Radiometer Medical ApS, Brønshøj, Denmark), } \\
\text { glicosímetro Precision PCx (Abbott Diabetes Care, } \\
\text { Amersfoort, The Netherlands) e sistema subcutâneo } \\
\text { contínuo da glicose (CGMS System Gold; Medtronic } \\
\text { MiniMed, Inc., Northridge, CA, USA). } \\
\text { Método de referência: laboratorial. } \\
\text { Tipo de amostra de sangue: arterial. }\end{array}$ & $\begin{array}{c}\text { Acurácia e confiabilidade: } \\
\text { O analisador de gases mostrou boa acurácia e precisão } \\
\text { comparado ao método de referência. O sistema contínuo } \\
\text { mostrou boa correlação quando comparado ao } \\
\text { laboratório. } \\
\text { Fatores relacionados ao manuseio do aparelho: } \\
\text { O glicosímetro Precision PCx mostrou confiabilidade } \\
\text { limitada do sangue e das fitas teste. } \\
\text { O sistema contínuo precisa ser calibrado } 4 \text { vezes ao dia e } \\
\text { os resultados não são imediatos, podendo estar } \\
\text { disponíveis após } 24 \text { horas, inviabilizando o uso na UTI. }\end{array}$ \\
\hline Karon et al. 2014(46); EUA, IV. & $\begin{array}{l}2695 \text { medidas, idade não } \\
\text { informada. }\end{array}$ & $\begin{array}{l}\text { Duas fases, retrospectivo e prospectivo. } \\
\text { Dispositivos utilizados: ACCU Check Inform (Roche } \\
\text { Diagnostics, Indianapolis, IN) e Nova StatStrip (Nova } \\
\text { Biomedical Waltham, MA). } \\
\text { Método de referência: laboratorial. } \\
\text { Tipo de amostra de sangue: } 95 \% \text { de cateter arterial e } \\
5 \% \text { de cateter venoso. }\end{array}$ & $\begin{array}{l}\text { Acurácia e confiabilidade: } \\
\text { O glicosímetro AccuChek Inform não cumpriu as diretrizes } \\
\text { de precisão do ISO. A precisão foi menor comparada ao } \\
\text { método de referência. O dispositivo Nova StatStrip } \\
\text { mostrou resultados com maior precisão de acordo ISO } \\
\text { 15197:2013. }\end{array}$ \\
\hline Shearer et al. $20099^{(47)}$, EUA, IV. & $\begin{array}{l}63 \text { pacientes, média de idade } 63,8 \\
\text { anos. }\end{array}$ & $\begin{array}{l}\text { Prospectivo. } \\
\text { Dispositivos utilizados: glicosímetro (Sure Step Flexx, } \\
\text { Johnson \& Johnson, Lifescan Inc, Milpitas, California) } \\
\text { Método de referência: laboratorial. } \\
\text { Tipo de amostra de sangue: capilar e venoso do CVC. }\end{array}$ & $\begin{array}{c}\text { Acurácia e confiabilidade: } \\
\text { O glicosímetro apresentou } 20 \% \text { dos valores diferentes do } \\
\text { laboratório. } \\
\text { Os valores laboratoriais diferiram significativamente entre } \\
\text { as amostras de sangue capilar e sangue do cateter. } \\
\text { Fatores relacionados ao paciente: } \\
\text { Não houve associação dos resultados obtidos pelo } \\
\text { glicosímetro com os níveis de hematócrito e pressão } \\
\text { arterial média. }\end{array}$ \\
\hline Cook et al. $2009^{(48)}$, EUA, IV. & $\begin{array}{l}67 \text { pacientes, média de idade } 58,4 \\
\text { anos. }\end{array}$ & $\begin{array}{l}\text { Prospectivo. } \\
\text { Dispositivo utilizado: glicosímetro (Sure Step Flexx, } \\
\text { Johnson \& Johnson LifeScan, Inc, Milpitas, California). } \\
\text { Método de referência: laboratorial. } \\
\text { Tipo de amostra de sangue: venoso do CVC e capilar. }\end{array}$ & $\begin{array}{c}\text { Acurácia e confiabilidade: } \\
\text { Os valores obtidos pelo glicosímetro mostraram } \\
\text { imprecisão quando comparados método de referência. } \\
\text { As medidas do glicosímetro mostraram até } 20 \mathrm{mg} / \mathrm{dL} \text { de } \\
\text { diferença entre os tipos de amostra de sangue. } \\
\text { Fatores relacionados ao paciente: } \\
\text { O nível do hematócrito diminuiu a precisão do } \\
\text { glicosímetro. }\end{array}$ \\
\hline
\end{tabular}




\begin{tabular}{|c|c|c|c|}
\hline $\begin{array}{l}\text { Autor, ano, País de procedência, } \\
\text { Nível de evidência }\end{array}$ & Amostra, Idade & $\begin{array}{l}\text { Delineamento do estudo, métodos de mensuração } \\
\text { da glicemia }\end{array}$ & Principais Resultados \\
\hline Mann et al. $2008^{(49)}$, EUA, IV. & $\begin{array}{l}195 \text { pacientes, idade não } \\
\text { informada. }\end{array}$ & $\begin{array}{c}\text { Coorte. } \\
\text { Dispositivos utilizados: Accu-Check Inform (Roche } \\
\text { Diagnostics, Indianapolis, IN); Accu Check Advantage } \\
\text { (Roche Diagnostics, Indianapolis, IN) e Medisense } \\
\text { Precision PCx (Abbot Diagnostics, Aboot Park, IL). } \\
\text { Método de Referência: laboratorial e glicosímetro } \\
\text { SureStep Flexx (LifeScan, Milpitas, CA) para estimar } \\
\text { taxas de erro. } \\
\text { Tipo de amostra de sangue: sangue arterial ou } \\
\text { venoso. }\end{array}$ & $\begin{array}{c}\text { Acurácia e confiabilidade: } \\
\text { As medidas dos glicosímetros foram elevados em } \\
\text { comparação com os valores laboratoriais. } \\
\text { Fatores relacionados ao paciente: } \\
\text { Os níveis de hematócrito influenciaram nas taxas de erro } \\
\text { dos glicosímetro. Fórmulas matemáticas podem corrigir } \\
\text { estes erros. }\end{array}$ \\
\hline Gijzen et al. 2012(50), EUA, IV. & $\begin{array}{c}80 \text { pacientes, média idade } 68,3,390 \\
\text { medidas de glicemia. }\end{array}$ & $\begin{array}{c}\text { Prospectivo, observacional. } \\
\text { Dispositivos utilizados: Accu Check Inform II System; } \\
\text { HemoCue Glu201DM, Nova StatStrip; Aboot Precision } \\
\text { Pro e o Menarini GlucoCard Memory PC. } \\
\text { Método de Referência: laboratorial. } \\
\text { Tipo de amostra de sangue: arterial. }\end{array}$ & $\begin{array}{c}\text { Acurácia e confiabilidade: } \\
\text { Os aparelhos Accu Check Inform, HemoCue e Precision } \\
\text { mostraram boa precisão e cumpriram os critérios ISO } \\
\text { 15197, os dispositivos NovaStat Strip e Menarini não } \\
\text { cumpriram os critérios. } \\
\text { Fatores relacionados ao paciente: } \\
\text { Nível de hematócrito, sódio, potássio, nitrogênio, } \\
\text { bilirrubina, proteína total, albumina não influenciaram nos } \\
\text { resultados de glicose. }\end{array}$ \\
\hline $\begin{array}{c}\text { Watkinson et al. 2012(51), Reino } \\
\text { Unido, III }\end{array}$ & $\begin{array}{c}206 \text { pacientes, média de idade } 56 \\
\text { anos. }\end{array}$ & $\begin{array}{l}\text { Ensaio clínico, prospectivo. } \\
\text { Dispositivos utilizados: glicosímetros Precision PC } \chi \\
\text { (Medisense glucose dehydrogenase, Maidenhead, UK) } \\
\text { e o HemoCue 201DM (glucose dehydrogenase, } \\
\text { Angelholm, Sweden); e o hemogasômetro } \\
\text { Radiometer } 700 \text { (glucose electrode) } \\
\text { Método de referência: laboratorial. } \\
\text { Tipo de amostra de sangue: arterial. }\end{array}$ & $\begin{array}{l}\text { Acurácia e confiabilidade: } \\
\text { HemoCue não apresentou diferenças com o método } \\
\text { laboratorial. } \\
\text { Os dois glicosímetros apresentaram ser imprecisos quando } \\
\text { comparado às análises do laboratório. } \\
\text { O dispositivo Radiometer teve desempenho similar ao } \\
\text { método laboratorial. } \\
\text { Fatores relacionados ao paciente: } \\
\text { A concentração total de glicose no sangue, os níveis de } \\
\text { hematócrito, pH e oxigênio, podem reduzir a precisão da } \\
\text { medida. }\end{array}$ \\
\hline
\end{tabular}

Legenda: UTI, Unidade de Terapia Intensiva; ISO, International Organization for Standartization, CVC - Cateter Venoso Central; CVP, Pressão venosa central; pH concentração hidrogeniônica. 


\section{DISCUSSÃO}

A produção científica encontrada sobre a confiabilidade e acurácia dos métodos de verificação da glicemia evidenciou os seguintes aspectos: método de mensuração e tipo de amostra, características do paciente crítico e manipulação do aparelho.

\section{Método de mensuração e tipo de amostra}

A análise dos artigos evidenciou $14(77,7 \%)$ estudos nos quais foram realizadas comparações entre os resultados da mensuração glicêmica obtidos com glicosímetro e a análise em laboratório (adotado por estes autores como método de referência) $)^{(34,36-37,39-41,43-50)}$. Quatro $(22,2 \%)$ estudos, cujos os métodos para comparação foram (glicosímetro e laboratório) foi referido que os valores foram superestimados pelo dispositivo portátil, mostrando discordância ${ }^{(41,44,46,49)}$.

Adicionalmente, dois $(11,1 \%)$ estudos consideraram o analisador de gases como método de referência ${ }^{(28,44)}$ e dois $(11,1 \%)$ utilizaram o glicosímetro portátil como padrão ouro ${ }^{(38,42)}$.

Em relação ao tipo de amostra de sangue os resultados mostraram que em seis $(33,3 \%)$ estudos utilizou-se venoso e capilar ${ }^{(36,39,43-44,47-48)}$, em quatro $(22,2 \%)$ arterial e capilar ${ }^{(35,37-38)}$, em dois $(11,1 \%)$ arterial e venoso $^{(46,49)}$, em quatro $(22,2 \%)$ somente arterial ${ }^{(34,38,43-44)}$, em um $(5,5 \%)$ arterial, venoso e capilar ${ }^{(34)}$ e em um $(5,5 \%)$ somente venoso proveniente de CVC e punção venosa ${ }^{(40)}$.

Destaca-se que os critérios ISO 15.197 estabelecem diretrizes para definição da acurácia do glicosímetro quando comparada aos resultados de análises laboratoriais. Os critérios publicados em 2003 indicam que $\geq 95 \%$ dos resultados da medição devem respeitar um valor máximo de intervalos de $\pm 15 \mathrm{mg} / \mathrm{dL}$ em concentrações de glicose $<75 \mathrm{mg} / \mathrm{dL}$, em concentrações de glicose superior à $75 \mathrm{mg} / \mathrm{dL}$, a margem de erro aceitável é transformada em porcentagem e ajustado a $15 \%$. Recomendações mais recentes ${ }^{(53)}$ propõem intervalos de $\pm 15 \mathrm{mg} / \mathrm{dL}$ em concentrações de glicose <100 mg / dL, e em concentrações à $100 \mathrm{mg} / \mathrm{dL}$, a margem de erro aceitável é transformada em porcentagem e ajustado a $20 \%$. Esses critérios nortearam sete $(38,8 \%)$ dos estudos, que mostraram desacordo com as recomendações $(35-38,41,46,50)$, e apenas um $(5,5 \%)$ cumpriu as normas.

A partir desses achados, acredita-se que, apesar do glicosímetro ser um método rápido, de fácil manipulação, que requer baixo volume amostral e com baixo custo, o benefício de uma verificação de glicemia utilizando-se estes aparelhos pode ser inferior ao de uma análise em laboratório. Dessa forma, tendo em vista o amplo uso de glicosímetro em vários serviços de saúde, sugere-se que os profissionais tenham atenção ao considerar medições com este dispositivo frente a resultados de glicemia muito baixos ou muito elevados. Nestes casos, é importante discutir com a equipe a possibilidade de checar essas medidas utilizando outro método, como o laboratorial e, também considerar as condições clínicas do paciente, as quais darão suporte à intervenções seguras no controle glicêmico.

Quanto ao sítio utilizado para coleta de sangue, um (5,5\%) estudo ${ }^{(39)}$ utilizou a orelha como local alternativo para obter sangue capilar, além da polpa digital, buscando avaliar se essa mudança implicaria em resultados mais seguros, no entanto, esse sítio amostral não melhorou a precisão do glicosímetro. Outros sete $(38,8 \%)$ estudos que utilizaram amostra de sangue capilar realizaram a coleta por punção em polpa digital $34,36-37,43,43,47-48)$. 
A presença de edema em pacientes críticos pode não refletir os níveis de glicose absoluta no sangue, já que em estado hipercatabólico, a proporção de glicose que alcança a periferia é baixa, tornando a glicemia de sangue capilar menos representativa do que ocorre nos compartimentos arteriais $^{(54)}$.

Sob outra perspectiva, estudos desaconselharam o uso de amostras de sangue obtidas por CVC devido a uma variação de até $20 \mathrm{mg} / \mathrm{dL}$ na glicemia quando comparada à amostras por punção venosa ou sangue arterial $(34,40,47)$.

Adicionalmente um (5,5\%) estudo verificou se poderia ocorrer contaminação de glicose exógena em amostras obtidas de diferentes locais e se isso poderia influenciar o resultado de valores glicêmicos, e, apesar dos seus achados mostrarem que não houve contaminação, eles revelaram diferenças entre valores glicêmicos resultantes da coleta por diferentes vias (CVC, PICC ou punção venosa) $)^{(40)}$. Pesquisadores afirmam que a via mais recomendada para obtenção de amostra em pacientes críticos é a arterial(20), seguida pela amostra colhida através do $\mathrm{CVC}^{(55)}$.

Os monitores subcutâneos contínuos de glicemia são considerados dispositivos eficazes para medir a glicemia devido à capacidade de fornecer informações sobre a direção, magnitude, duração, frequência de flutuações dos níveis de glicose do sangue ${ }^{(56)}$.

Esses dispositivos foram analisados em três $(16,6 \%)$ estudos $(35,42,45)$, os quais indicaram resultados divergentes. Entre eles o estudo realizado em Amsterdã em 2012 ${ }^{(28)}$ mostrou que podem ocorrer problemas técnicos como pedidos de calibração extra pelo equipamento, erro na leitura da glicemia quando as medições se iniciam antes da calibragem do aparelho, retirada prematura dos sensores devido alarmes/ruídos que reduzem a confiabilidade e a acurácia desse tipo de dispositivo. Nesse mesmo estudo o dispositivo foi considerado mais confiável nos indivíduos diabéticos, pois os resultados mostraram-se mais próximos quando comparados ao método de referência do estudo (hemogasômetro).

Price et al (2008), referem que o monitor contínuo não é preciso, em comparação com o glicosímetro(42). No estudo de Corstjens et al (2006) as medidas do monitor contínuo da glicemia também não foram acuradas e confiáveis quando comparadas as medidas do glicosímetro, mas mostraram-se similares aos resultados no laboratório(42,45).

Recentemente, um ensaio clínico avaliou a precisão, viabilidade e aceitação pela equipe de enfermagem de um monitor subcutâneo contínuo de glicemia em 20 UTIs. Os resultados mostraram que a acurácia desse dispositivo foi reduzida quando houve maior amplitude dos resultados de glicemia, ou seja, ele não mediu com precisão níveis baixos ou elevados de glicose ${ }^{(18)}$.

A literatura traz estudos que evidenciaram que alguns fármacos como acetaminofeno, ácido ascórbico podem interferir nos resultados de glicemia obtidos por monitores contínuos ${ }^{(57-58)}$. Algumas substâncias como a maltose, xilose e a icodextrina também alteraram as leituras do monitor contínuo, sendo que a última foi responsável por aumentos no valor de glicose em mais de $100 \mathrm{mg} / \mathrm{d}^{(59)}$. Outra limitação para o uso de monitores seria a imprecisão das medidas nas primeiras 24 horas após a inserção do sensor subcutâneo decorrente da inflamação tecidual local(60), portanto, estes resultados mostram que os sistemas de monitoração contínua requerem mais testes para avaliar a viabilidade de seu uso. 
A Food and Drug Administration (FDA) recomenda que as doses de insulina sejam baseadas em valores de glicose no sangue e não em monitores contínuos, por estes utilizarem amostra intersticial. Perante esses resultados, o monitor contínuo de glicemia parece ser uma inovação sofisticada e promissora no controle glicêmico, mas ainda não há estudos suficientes que o respaldem em relação à acurácia e à confiabilidade do seu uso em pacientes críticos, além disso, estima-se que o seu custo seja mais elevado tornando-o inacessível financeiramente à maioria das UTIs brasileiras.

Vale ressaltar que novas tecnologias de monitorização contínua da glicemia estão sendo inseridas na rotina das UTIs, como os monitores contínuos de glicemia intravenosa ou por espectroscopia, nos quais o dispositivo utilizado a beira leito é acoplado a uma linha proximal do CVC que apresente retorno venoso, e após obtenção, centrifugação do sangue a medida é analisada por uma tecnologia de luz infravermelha e então apresentado o resultado. Esses monitores foram considerados seguros e precisos para o cuidado de pacientes críticos $^{(61-63)}$.

Esta revisão mostrou ainda que em dois $(11,1 \%)$ estudos que testaram o analisador de gás sanguíneo (hemogasômetro) comparando-o com outros métodos com monitor subcutâneo contínuo ou com o glicosímetro ${ }^{(46,60)}$ mostraram resultados confiáveis. Um estudo de revisão corrobora esses resultados ${ }^{(20)}$.

Acrescendo essas conclusões, um outro estudo mostra comparações entre os testes laboratoriais e o analisador de gases sanguíneos na verificação da glicemia, e destaca que a precisão é similar entre os dois métodos e considerando o tempo de resposta, o volume da amostra e o custo, o hemogasômetro parece ser mais vantajoso. O mesmo estudo demonstra ainda que parece não haver diferença no custo por teste entre esse dispositivo e os dispositivos portáteis ${ }^{(16)}$.

Ressalta-se que esse aparelho, tanto quanto os outros métodos, requer cuidados com a sua manutenção, e para o uso dos analisadores de gases sanguíneos, a equipe da UTI deve ser treinada, o que pode demandar mais tempo para obter uma amostra (sangue arterial ou venoso) e direcioná-la ao aparelho para obtenção de resultados, o que provavelmente implicaria em aumento da carga de trabalho, gerando mais custos ao serviço.

Ainda assim, presume-se que o uso dos analisadores de gases sanguíneos seja um método confiável e propicie segurança aos pacientes críticos, pois é possível que estes sejam alocados dentro ou próximos das UTIs, além de serem capazes de fornecer apoio às demais condutas da equipe por produzirem outros parâmetros (níveis de $\mathrm{pH}, \mathrm{PaCO}_{2}$, potássio e sódio) bastante úteis em UTI.

\section{Fatores relacionados às características do paciente crítico}

Em relação às características do paciente crítico, quatro $(22,2 \%)$ estudos ${ }^{(43-44,48-49)}$ mostraram que níveis alterados de hematócrito podem afetar a precisão dos valores de glicemia nas comparações entre o glicosímetro e a análise realizada em laboratório, resultado que não foi observado em três (16,6\%) estudos ${ }^{(47,50-51)}$.

Os valores normais de hematócrito variam de $37 \%$ a $47 \%$ em mulheres e de $42 \%$ a $52 \%$ em homens, nesse sentido os valores de hematócrito inferiores ao normal resultam em superestimação do resultado de glicemia, devido a diminuição de glóbulos vermelhos que leva a uma redução do deslocamento de plasma, resultando em moléculas com mais glicose disponível para reação com a enzima contida na tira (fita) de mensuração de glicose ${ }^{(57)}$. 
Ainda levando em consideração o papel do hematócrito na acurácia e confiabilidade da análise da glicemia, um $(5,5 \%)$ estudo ${ }^{(49)}$ propõe fórmulas matemáticas para cada aparelho para reduzir as taxas de erros decorrentes dos níveis de hematócrito.

Um estudo (5,5\%) destacou o edema como uma condição associada à imprecisão do resultado dos níveis de glicemia ${ }^{(43)}$. Dois $(11,1 \%)$ avaliaram ainda se os gases sanguíneos $\left(\mathrm{pO}^{2}\right)$ e o pH poderiam afetar o resultado da mensuração da glicemia ${ }^{(41,50)}$, todavia não encontraram interferência. Ginsberg (2009), mostrou uma preocupação nesse sentido, pois as tiras teste dos glicosímetros são geralmente calibradas para concentrações de oxigênio capilar, e valores elevados de oxigênio como os encontrados em amostras arteriais ou em pacientes que recebem oxigênio, podem resultar em valores de glicemia menores, assim como baixos níveis de oxigênio, como os encontrados em amostras venosas ou em pacientes com grave doença pulmonar obstrutiva crônica podem causar valores falsamente altos $^{(64)}$. O estudo de Eastham et al., (2009) demonstrou que os níveis de bilirrubina, galactose, hematócrito, triglicerídeos e o ácido úrico também interferiram na precisão da mensuração glicêmica ${ }^{(65)}$.

A administração de vasopressores (catecolaminas) é comum em pacientes críticos hemodinamicamente instáveis, e o uso deste fármaco foi associado a maior chance de erro na medida na amostra capilar em 3 (16,6\%) estudos $^{(34,36,38)}$. Conforme a literatura, outros fármacos como os sedativos comumente utilizados em pacientes críticos (que induzem o bloqueio dos beta-adrenorreceptores) ${ }^{(66)}$ e o ácido ascórbico (vitamina C) ${ }^{(67)}$ também podem mascarar os resultados da glicemia.

Ainda que os fatores supracitados possam variar conforme o paciente, considera-se que a equipe deve ter cautela ao se deparar com valores de glicemia extremos. Para a definição de intervenções, a equipe deve conhecer e avaliar condições clínicas do paciente, tais como: níveis de hematócrito, parâmetros respiratórios $\left(\mathrm{pH}, \mathrm{pCO}{ }^{2}\right)$, estabilidade hemodinâmica (pressão arterial) e neurológicas e ponderar sobre a confirmação e/ou verificação da glicemia por meio de outro método, como o laboratorial ou o hemogasômetro.

\section{Fatores relacionados à manipulação do aparelho}

Dois (11,1\%) estudos que abordaram os fatores relacionados à manipulação enfocaram especificamente no aparelho glicosímetro, citando que o número de calibrações, o uso de vários lotes de fitas, lancetas e os tipos de aparelhos utilizados em uma mesma unidade ${ }^{(45,50)}$ podem interferir na acurácia dos resultados de glicemia. Esses resultados são similares aos de um estudo realizado em um departamento de emergência, em que se recomendou o "reteste" em no máximo 10 minutos pelo mesmo profissional e utilizando o mesmo medidor ao deparar-se com valores de glicemia críticos, objetivando aumento da precisão(68).

Outros estudos corroboram essas afirmações e dizem que as condições de armazenamento das fitas teste, incluindo a umidade e a temperatura do ambiente, a desinfecção do glicosímetro após cada uso quando utilizado em múltiplos pacientes ${ }^{(70-72)}$ e a calibração dos aparelhos (glicosímetro, analisador de gás sanguíneo, laboratório) podem interferir nos resultados obtidos.

No caso dos dispositivos portáteis, supõe-se que o contato destes com superfícies contaminadas, com sujidades ou o excesso de manipulação das fitas que atuam por reações enzimáticas (glicose oxidase, peroxidase, hexoquinase) pode gerar confundidores interagindo com essas enzimas ${ }^{(42)}$ e interferir nos resultados de glicemia (interpretados pelos métodos fotométrico ou amperométrico). 
Vale ressaltar que, muitas vezes na prática clínica não há norma de padronização de vias de coleta, permitindo a equipe de enfermagem escolher o local para punção conforme sua preferência, utilizando nessa situação variados sítios de coleta para um mesmo paciente o que pode levar a erros de interpretação; assim como a qualidade das lancetas utilizadas, pois em algumas instituições a escolha desta se dá pelo menor custo, no entanto, nem sempre trarão melhor benefício ao paciente.

Além disso, as unidades podem não dispor de lancetas e utilizam agulhas de menor calibre para realização da punção capilar. Enfatiza-se que não há legislação específica que trate sobre o material ideal para punção de sangue capilar nas UTIs, contudo uma das normas regulamentadoras (NR) dos serviços de saúde, a 32, indica uso sistemático de dispositivos de segurança e material mais adequado de acordo com a prática a ser realizadaa ${ }^{(72)}$.

Casualmente, um pesquisador descobriu que a coleta de sangue capilar realizada após o contato das mãos do indivíduo com doces, biscoitos ou frutas podem gerar valores de glicemia falsamente elevados, sendo que essa contaminação pode ser uma fonte importante de imprecisão. $O$ uso de loção e sabonete também foi testado, entretanto não alterou a precisão do aparelho(64).

Pondera-se que o ato de higienização das mãos pelos profissionais é essencial e amplamente difundido na prevenção de infeç̧ão, porém avaliando os achados de Ginsberg (2009), destaca-se a importância desse ato também na verificação da glicemia, pois a lavagem incorreta das mãos ou a negligência deste ato poderiam interferir na acurácia e confiabilidade dos resultados de glicemia ${ }^{(64)}$.

Alguns estudos relatam que sobrecarga de trabalho da equipe de enfermagem pode favorecer uma assistência insegura relacionada à ocorrência de infecções, quedas, e erros de medicação(73), entretanto, é necessária a realização de estudos para sustentar associações entre a carga de trabalho da enfermagem e a acurácia e confiabilidade nas mensurações glicêmicas.

Estima-se que na prática clínica, os fatores como o local de coleta da amostra e tipo de método utilizado para mensuração podem ser mesclados em um mesmo turno de trabalho e para um mesmo paciente. Além disso, essas condições também são afetadas pela conduta clínica de cada profissional, pelo desconhecimento da equipe sobre os fatores que favorecem a imprecisão e a segurança, pelo julgamento crítico, pelo raciocínio clínico, pela evolução clínica dos pacientes e o prognóstico, pelo uso de glicosímetros de marcas diferentes em uma mesma unidade e pela falta de normas de procedimentos que respaldem as escolhas. Em síntese, esse dissenso na prática pode acarretar em confusão na interpretação sobre a evolução do quadro glicêmico.

Elucida-se a importância deste estudo com propósito de conhecer os fatores que interferem na medida da glicemia para garantir a acurácia e a confiabilidade dos resultados, além da segurança ao paciente crítico. Entretanto, evidencia-se como uma limitação, a falta de consenso para recomendação de alguns métodos de mensuração, fato que, na atividade clínica poderia equivocar a equipe, contudo, estudos futuros podem averiguar o conhecimento da equipe que assiste ao paciente crítico em UTIs e investigar de que maneira ocorre a manejo destes elementos de interferência da medida na prática, considerando distintos cenários.

\section{CONSIDERAÇÕES FINAIS}

A acurácia e a confiabilidade da mensuração da glicemia em pacientes críticos, está relacionada aos dispositivos utilizados para a medida, às características clínicas do doente (níveis de hematócrito, de gases 
arteriais que refletem os parâmetros respiratórios e metabólicos do paciente, estabilidade hemodinâmica) e ao manuseio dos aparelhos que podem interferir nos resultados dos medidores. Esta revisão recomenda que a equipe que atua em UTI, esteja instruída e atenta às necessidades e características clínicas dos pacientes, aos métodos disponíveis nos serviços de saúde bem como suas vantagens e limitações e pondere sobre qual a melhor escolha, considerando a sua realidade e a segurança do paciente.

\section{REFERÊNCIAS}

1. Dungan KM, Braithwaite SS, Preiser JC. Stress hyperglycaemia. Lancet [Internet]. 2009. [acesso em: 15 abr. 2017]; 23;373(9677):1798-807. Disponível em: http://doi.org/10.1016/S0140-6736(09)60553-5.

2. Marik PE, Bellomo R. Stress hyperglycemia: an essential survival response!. Critical Care [Internet]. 2013. [acesso em: 15 abr. 2017];17(2):305. Disponível em: http://doi.org/10.1186/cc12514.

3. Finfer S, Liu B, Chittock DR, Norton R, Myburgh JA, McArthur C, et al. Hypoglycemia and risk of death in critically ill patients. N Engl J Med [Internet]. 2012. [acesso em: 15 abr. 2017];367(12):1108-18. Disponível em: http://doi.org/10.1056/NEJMoa1204942. 4. Egi M, Bellomo R, Stachowski E, French CJ, Hart GK, Hegarty C, et al. Blood glucose concentration and outcome of critical illness: the impact of diabetes. Crit Care Med [Internet]. 2008. [acesso em: 15 abr. 2017]; 36(8):2249-55. Disponível em: http://doi.org/10.1097/CCM.0b013e318181039a.

5. Viana MV, Moraes RB, Fabbrin AR, Santos MF, Gerchman F. Assessment and treatment of hyperglycemia in critically ill patients. Rev Bras Ter Intensiva [Internet]. 2014. [acesso em: 15 abr. 2017];26(1):71-6. Disponível em: http://doi.org/10.5935/0103507X.20140011.

6. Vanhorebeek I, Langouche L, Van den Berghe G. Tight blood glucose control with insulin in the ICU: facts and controversies. Chest [Internet]. 2007. [acesso em: 15 abr. 2017];132(1):268-78. Disponível em: http://doi.org/10.1378/chest.06-3121.

7. Montori VM, Bistrian BR, McMahon MM. Hyperglycemia in Acutely III Patients. JAMA [Internet]. 2002. [acesso em: 15 abr. 2017];288(17):2167-9. Disponível em: http://doi.org/10.1001/jama.288.17.2167.

8. Langouche L, Van den Berghe G. Glucose metabolism and insulin therapy. Crit Care Clin [Internet]. 2006. [acesso em: 15 abr. 2017];22(1):119-29, vii. Disponível em: http://doi.org/10.1016/j.ccc.2005.09.005.

9. Turina M, Fry DE, Polk HC, Jr. Acute hyperglycemia and the innate immune system: clinical, cellular, and molecular aspects. Crit Care Med [Internet]. 2005. [acesso em: 15 abr. 2017];33(7):1624-33. Disponível em:

http://doi.org/10.1097/01.CCM.0000170106.61978.D8.

10. Van Den Berghe G, Wouters $P$, Weekers F, Verwaest C, et al. Intensive insulin therapy in critically ill patients. N Engl J Med [Internet]. 2001. [acesso em: 15 abr. 2017]; 345(19):1359-67. Disponível em: http://doi.org/10.1056/NEJMoa011300.

11. Umpierrez GE, Hellman R, Korytkowski MT, Kosiborod M, Maynard GA, et al. Management of hyperglycemia in hospitalized patients in non-critical care setting: an endocrine society clinical practice guideline. J Clin Endocrinol Metab [Internet]. 2012.

[acesso em: 15 abr. 2017] Jan;97(1):16-38. Disponível em: http://doi.org/10.1210/jc.2011-2098.

12. Sauer P, Van Horn ER. Impact of intravenous insulin protocols on hypoglycemia, patient safety, and nursing workload. Dimens Crit Care Nurs [Internet]. 2009. [acesso em: 15 abr. 2017];28(3):95-101. Disponível em:

http://doi.org/10.1097/DCC.0b013e31819af06d.

13. Van den Berghe G, Wilmer A, Hermans G, Meersseman W, Wouters PJ, et al. Intensive insulin therapy in the medical ICU. $N$ Engl J Med [Internet]. 2006. [acesso em: 10 abr. 2017]; Feb 2;354(5):449-61. Disponível em:

http://doi.org/10.1056/NEJMoa052521.

14. Egi M, Finfer S, Bellomo R. Glycemic control in the ICU [Internet] 2011. [acesso em: 10 abr. 2017];140(1):212-220. Disponível em: http://doi.org/10.1378/chest.10-1478.

15. Stamou SC, Nussbaum M, Carew JD, Skipper E, Robicsek F, Lobdellet KW. Hypoglycemia with intensive insulin therapy after cardiac surgery: predisposing factors and association with mortality. J Thorac Cardiovasc Surg. [Internet]. 2011. Jul [acesso em: 10 abr. 2017];142(1):166-73. Disponível em: http://doi.org/10.1016/i.jtcvs.2010.09.064.

16. Le HT, Harris NS, Estilong AJ, Olson A, Rice MJ. Blood glucose measurement in the intensive care unit: what is the best method? J Diabetes Sci Technol [Internet]. 2013. [acesso em: 10 abr. 2017];7(2):489-99. Disponível em:

http://doi.org/10.1177/193229681300700226.

17. Schierenbeck F, Franco-Cereceda, Liska J. J Diabetes Sci Technol. [Internet]. 2012. [acesso em: 10 abr. 2017]; 6(6): $1365-1371$. Disponível em: http://doi.org/10.1177/193229681200600615.

18. Wollersheim T, Engelhardt LJ, Pachulla J, Moergeli R, Koch S, Spies C, et al. Accuracy, reliability, feasibility and nurse acceptance of a subcutaneous continuous glucose management system in critically ill patients: a prospective clinical trial. Ann Intensive Care. 2016. [Internet] [acesso em: 10 abr. 2017];6(1):70. Disponível em: http://doi.org/10.1186/s13613-016-0167-z.

19. Rajendran R, Rayman G. J Diabetes Sci Technol. [Internet] 2014. [acesso em: 10 abr. 2017]; 8(6): 1081-1090. Disponível em: http://doi.org/10.1177/1932296814538940. 
20. Inoue S, Egi M, Kotani J, Morita K. Accuracy of blood-glucose measurements using glucose meters and arterial blood gas analyzers in critically ill adult patients: systematic review. Crit Care [Internet] 2013. [acesso 06 abr 2017]; 17(2): R48. Disponível em: http://doi.org/10.1186/cc12567.

21. Tonyushkina K, Nichols JH. Glucose meters: a review of technical challenges to obtaining accurate results. J Diabetes Sci Technol. 2009. [Internet] [acesso em: 10 abr. 2017]; 1;3(4):971-80. Disponível em: http://doi.org/10.1177/193229680900300446. 22. Klonoff DC. Point-of-Care Blood Glucose Meter Accuracy in the Hospital Setting. Diabetes Spectr [Internet]. 2014. [acesso em: 10 abr. 2017]; 27(3): 174-179. Disponível em: http://doi.org/10.2337/diaspect.27.3.174.

23. Monteiro SCM, Gomes E, Belfort IK, Avelar MF, Sampaio RM. Análise comparativa da determinação de glicemia capilar e venosa com glicosímetro versus dosagem laboratorial. Rev Pesq Saúde. 2015; 16(1): 41-44. Disponível em:

http://www.periodicoseletronicos.ufma.br/index.php/revistahuufma/article/view/4075.

24. Preiser JC, van Zanten ARH, Berger MM, Biolo G, Casaer MP, et al. Metabolic and nutritional support of critically ill patients: consensus and controversies. Crit Care [Internet]. 2015. [acesso em: 10 abr. 2017]; 19(1): 35. Disponível em:

http://doi.org/10.1186/s13054-015-0737-8.

25. Paixão CT, Nepomuceno RM, Santos MM, Silva LD. Fatores predisponentes para hipoglicemia: aumentando a segurança do paciente crítico que utiliza insulina intravenosa. Rev enferm UERJ [Internet] 2015. [acesso em: 10 abr. 2017]; 23(1):70-5. Disponível em: http://dx.doi.org/10.12957/reuerj.2015.15098.

26. Galvão CM, Sawada NO, Rossi LA. A prática baseada em evidências: considerações teóricas para sua implementação na enfermagem perioperatória. Rev. Latino-Am. Enfermagem [Internet]. 2002. [cited 2017 Oct 07]; 10(5): 690-695. Disponível em: http://dx.doi.org/10.1590/S0104-11692002000500010.

27. Melnyk BM, Fineout-Overholt E, Stillwell SB, Williamson KM. Evidence-based practice: step by step: the seven steps of evidence-based practice. Am J Nurs [Internet]. 2010. [acesso em: 10 abr. 2017]; 110(1):51-3.

28. Santos CMC, Pimenta CAM, Nobre MRC. The PICO strategy for the research question construction and evidence search. Rev. Latino-Am. Enfermagem[Internet]. 2007. [acesso em: 10 abr. 2017]; 15 (3): 508-511.

29. Ursi ES, Galvão CM. Prevenção de lesões de pele no perioperatório: revisão integrativa da literatura. Rev. Latino-Am Enfermagem [Internet]. 2006. [acesso em: 10 abr. 2017] ;14 (1): 124-131. Disponível em: http://dx.doi.org/10.1590/S010411692006000100017.

30. Corl, Dawn. Quality control considerations for point of care blood glucose meters. Quality Control: Developments, Methods and Applications. 2012; 69-84.

31. Betz JM, Brown PN, Roman MC. Accuracy, precision, and reliability of chemical measurements in natural products research. Fitoterapia [Internet]. 2011. [acesso em: 18 abr. 2017]; 82(1):44-52. Disponível em: http://doi.org/10.1016/j.fitote.2010.09.011. 32. MELNYK BM; FINEOUT-OVERHOLT E. Evidence-based practice in nursing and healthcare: A guide to best practice. Philadelphia: Lippincott, Williams \& Wilkins. 2011.

33. Moher D, Shamseer L, Clarke M, Davina G, Liberati A, Petticrew M, Shekelle P, Stewart LA. Preferred reporting items for systematic review and meta-analysis protocols (PRISMA-P) 2015 statement. BMJ [Internet]. 2015. [acesso em: 18 abr. 2017]; 349:g7647. Disponível em: http://doi.org/10.1136/bmi.g7647.

34. Pereira AJ, Corrêa TD, de Almeida FP, Deliberato RO, Lobato MdS, Akamine N, et al. Inaccuracy of Venous Point-of-Care Glucose Measurements in Critically III Patients: A Cross-Sectional Study. PLoS ONE [Internet] 2015. [acesso em: 18 abr. 2017];10(6): e0129568. Disponível em: http://doi.org/10.1371/journal.pone.0129568.

35. Van Hooijdonk RT, Leopold JH, Winters T, Binnekade JM, Juffermans NP, Horn J, et al. Point accuracy and reliability of an interstitial continuous glucose-monitoring device in critically ill patients: a prospective study. Crit Care [Internet]. 2015. [acesso em: 18 abr. 2017];19:34. Disponível em: http://doi.org/10.1186/s13054-015-0757-4.

36. Garingarao CJ, Buenaluz-Sedurante M, Jimeno CA. Accuracy of point-of-care blood glucose measurements in critically ill patients in shock. J Diabetes Sci Technol [Internet]. 2014. [acesso em: 18 abr. 2017]; 8(5):937-44. Disponível em: http://doi.org/10.1177/1932296814538608.

37. Lonjaret L, Claverie V, Berard E, Riu-Poulenc B, Geeraerts T, Genestal M, et al. Relative accuracy of arterial and capillary glucose meter measurements in critically ill patients. Diabetes Metab [Internet]. 2012. [acesso em: 18 abr. 2017]; 38(3):230-5. Disponível em: http://doi.org/10.1016/j.diabet.2011.12.003.

38. Juneja D, Pandey R, Singh O. Comparison between arterial and capillary blood glucose monitoring in patients with shock. Eur J Intern Med [Internet]. 2011. [acesso em: 18 abr. 2017];22(3):241-4. Disponível em: http://doi.org/10.1016/j.ejim.2011.01.004. 39. Fekih Hassen M, Ayed S, Gharbi R, Ben Sik Ali H, Marghli S, Elatrous S. Bedside capillary blood glucose measurements in critically ill patients: influence of catecholamine therapy. Diabetes Res Clin Pract [Internet]. 2010. [acesso em: 18 abr. 2017]; 87(1):87-91. Disponível em: http://doi.org/10.1016/i.diabres.2009.09.018.

40. Karon BS, Koch CD, Wockenfus AM, Brown JK. Accuracy of whole blood glucose measurement when venous catheter blood samples are used on glucose meters. Diabetes Technol Ther [Internet]. 2009. [acesso em: 18 abr. 2017];11(12):819-25. Disponível em: http://doi.org/10.1089/dia.2009.0074. 
41. Hoedemaekers CW, Klein Gunnewiek JM, Prinsen MA, Willems JL, Van der Hoeven JG. Accuracy of bedside glucose measurement from three glucometers in critically ill patients. Crit Care Med [Internet]. 2008. [acesso em: 18 abr. 2017]; 36(11):3062-6. Disponível em: http://doi.org/10.1097/CCM.0b013e318186ffe6.

42. Price GC, Stevenson K, Walsh TS. Evaluation of a continuous glucose monitor in an unselected general intensive care population. Crit Care Resusc. 2008;10(3):209-16.

43. Critchell CD, Savarese V, Callahan A, Aboud C, Jabbour S, Marik P. Accuracy of bedside capillary blood glucose measurements in critically ill patients. Intensive Care Med [Internet]. 2007. [acesso em: 18 abr. 2017];33(12):2079-84. Disponível em:

http://doi.org/10.1007/s00134-007-0835-4.

44. Lacara T, Domagtoy C, Lickliter D, Quattrocchi K, Snipes L, Kuszaj J, et al. Comparison of point-of-care and laboratory glucose analysis in critically ill patients. Am J Crit Care. 2007;16(4):336-46.

45. Corstjens AM, Ligtenberg JJ, van der Horst IC, Spanjersberg R, Lind JS, Tulleken JE, et al. Accuracy and feasibility of point-of-care and continuous blood glucose analysis in critically ill ICU patients. Crit Care [Internet]. 2006. [acesso em: 18 abr. 2017]; $10(5)$ : R135. Disponível em: http://doi.org/10.1186/cc5048.

46. Karon BS, Blanshan CT, Deobald GR, Wockenfus AM. Retrospective evaluation of the accuracy of Roche AccuChek Inform and Nova StatStrip glucose meters when used on critically ill patients. Diabetes Technol Ther [Internet]. 2014. [acesso $07 \mathrm{abr}$ 2017];16(12):828-32. Disponível em: http://doi.org/10.1089/dia.2014.0074.

47. Shearer A, Boehmer M, Closs M, Dela Rosa R, Hamilton J, Horton K, et al. Comparison of glucose point-of-care values with laboratory values in critically ill patients. Am J Crit Care [Internet]. 2009. [acesso em: 18 abr. 2017]; 18(3):224-30. Disponível em: http://doi.org/10.4037/ajcc2009448.

48. Cook A, Laughlin D, Moore M, North D, Wilkins K, Wong G, et al. Differences in glucose values obtained from point-of-care glucose meters and laboratory analysis in critically ill patients. Am J Crit Care [Internet]. 2009. [acesso em: 18 abr. 2017]; 18(1):6571. Disponível em: http://doi.org/10.4037/ajcc2009626.

49. Mann EA, Salinas J, Pidcoke HF, Wolf SE, Holcomb JB, Wade CE. Error rates resulting from anemia can be corrected in multiple commonly used point-of-care glucometers. J Trauma [Internet]. 2008. [acesso em: 18 abr. 2017];64(1):15-20. Disponível em: http://doi.org/10.1097/TA.0b013e318160b9e4.

50. Gijzen K, Moolenaar DL, Weusten JJ, Pluim HJ, Demir AY. Is there a suitable point-of-care glucose meter for tight glycemic control? Evaluation of one home-use and four hospital-use meters in an intensive care unit. Clin Chem Lab Med [online]. 2012. [acesso em: 18 abr. 2017];50(11):1985-92.Disponível em: http://doi.org/10.1515/cclm-2012-0104.

51. Watkinson PJ, Barber VS, Amira E, James T, Taylor R, Young JD. The effects of precision, haematocrit, pH and oxygen tension on point-of-care glucose measurement in critically ill patients: a prospective study. Ann Clin Biochem [Internet]. 2012 [acesso em: 18 abr. 2017];49(Pt 2):144-51. Disponível em: http://doi.org/10.1258/acb.2011.011162.

52. International Organization for Standardization. In Vitro diagnostic test systems-requirements for blood-glucose monitoring systems for self-testing in managing diabetes mellitus. EN ISO 15197:2003.

53. International Organization for Standardization. In vitro diagnostic test systems-requirements for blood-glucose monitoring systems for self-testing in managing diabetes mellitus. ISO 15197:2013 (E).

54. Fahy BG, Coursin DB. Critical glucose control: the devil is in the details. Mayo Clin Proc [Internet]. 2008 [acesso em: 24 abr. 2017];83(4):394-7. Disponível em: http://doi.org/10.4065/83.4.394.

55. Finfer S, Wernerman J, Preiser JC, Cass T, Desaive T, Hovorka R, et al. Clinical review: Consensus recommendations on measurement of blood glucose and reporting glycemic control in critically ill adults. Crit Care [Internet]. 2013. [acesso em: 24 abr. 2017];17(3):229. Disponível em: http://doi.org/10.1186/cc12537.

56. Khadilkar KS, Bandgar T, Shivane V, Lila A, Shah N. Current concepts in blood glucose monitoring. Indian J Endocrinol Metab [online]. 2013. [acesso em: 24 abr. 2017]; p. S643-9. Disponível em: http://doi.org/10.4103/2230-8210.123556.

57. Heinemann L. Quality of Glucose Measurement with Blood Glucose Meters at the Point-of-Care: Relevance of Interfering Factors. Diabetes Technol Ther. [Internet]; 2010. [acesso em: 24 abr. 2017];12(11):847-57. Disponível em: http://doi.org/10.1089/dia.2010.0076.

58. Basu A, Veettil S, Dyer R, Peyser T, Basu R. Direct Evidence of Acetaminophen Interference with Subcutaneous Glucose Sensing in Humans: A Pilot Study. Diabetes Technol Ther [Internet]. 2016. [acesso em: 24 abr. 2017]; S2-43-7. Disponível em:

http://doi.org/10.1089/dia.2015.0410.

59. Schleis TG. Interference of maltose, icodextrin, galactose, or xylose with some blood glucose monitoring systems.

Pharmacotherapy [Internet]. 2007. [acesso em: 24 abr. 2017];27(9):1313-21. Disponível em:

http://doi.org/10.1592/phco.27.9.1313.

60. Rebrin K, Sheppard NF, Jr., Steil GM. Use of subcutaneous interstitial fluid glucose to estimate blood glucose: revisiting delay and sensor offset. J Diabetes Sci Technol. 2010. [acesso em: 24 abr. 2017]; 4(5):1087-98. Disponível em:

http://doi.org/10.1177/193229681000400507.

61. Bochicchio GV, Nasraway S, Moore L, Furnary A, Nohra E, Bochicchio K. Results of a multicenter prospective pivotal trial of the first inline continuous glucose monitor in critically ill patients. J Trauma Acute Care Surg. [Internet]. 2017. [acesso em: 30 dez 2016]. Disponível em: https://www.ncbi.nlm.nih.gov/pubmed/28328679. 
62. Nohra E, Buckman S, Bochicchio K, Chamieh J, Reese S, Merrill C, et al. Results of a near continuous glucose monitoring technology in surgical intensive care and trauma. Contemp Clin Trials [Internet]. 2016. [acesso em: 30 dez 2016];50:1-4. Disponível em: https://doi.org/10.1016/j.cct.2016.07.007.

63. Schierenbeck F, Franco-Cereceda A, Liska J. Accuracy of 2 Different Continuous Glucose Monitoring Systems in Patients Undergoing Cardiac Surgery. Journal of Diabetes Science and Technology. 2016. [acesso em: 30 dez 2016]; 11(1): 108-116. Disponível em: http://doi.org/10.1177/1932296816651632.

64. Ginsberg BH. Factors Affecting Blood Glucose Monitoring: Sources of Errors in Measurement. J Diabetes Sci Technol [Internet]. 2009. [acesso em: 30 dez 2016]; 3(4): 903-913. Disponível em: http://doi.org/10.1177/193229680900300438.

65. Eastham JH, Mason D, Barnes DL, Kollins J. Prevalence of interfering substances with point-of-care glucose testing in a community hospital. Am J Health Syst Pharm [Internet]. 2009. [acesso em: 30 dez 2016];66(2):167-70. Disponível em: http://doi.org/10.2146/ajhp070512.

66. Kavanagh BP, McCowen KC. Clinical practice. Glycemic control in the ICU. N Engl J Med [Internet]. 2010. [acesso em: 30 dez 2016]; 363(26):2540-6. Disponível em: http://doi.org/10.1056/NEJMcp1001115.

67. Lyon ME, DuBois JA, Fick GH, Lyon AW. Estimates of Total Analytical Error in Consumer and Hospital Glucose Meters Contributed by Hematocrit, Maltose, and Ascorbate. J Diabetes Sci Technol [Internet]. 2010. [acesso em: 30 abr 2017]; 63(26):2540-6. Disponível em: http://doi.org/10.1056/NEJMcp1001115.

68. Schifman RB, Nguyen TT, Page ST. Reliability of point-of-care capillary blood glucose measurements in the critical value range. Arch Pathol Lab Med [Internet]. 2014. [acesso em: 30 abr 2017];138(7):962-6. Disponível em: http://doi.org/10.5858/arpa.20130455-OA.

69. Haller MJ, Shuster JJ, Schatz D, Melker RJ. Adverse impact of temperature and humidity on blood glucose monitoring reliability: a pilot study. Diabetes Technol Ther [online]. 2007. [acesso em: 30 abr 2017];9(1):1-9. Disponível em:

http://doi.org/10.1089/dia.2006.0051.

70. Burnett RW, Covington AK, Fogh-Andersen N, Kulpmann WR, Maas AH, Muller-Plathe O, et al.: International Federation of Clinical Chemistry (IFCC). Scientific Division. Committee on $\mathrm{pH}$, Blood Gases and Electrolytes. Approved IFCC recommendations on whole blood sampling, transport and storage for simultaneous determination of $\mathrm{pH}$, blood gases and electrolytes. Eur $\mathrm{J}$ Clin $\mathrm{Chem}$ Clin Biochem 1995, 33:247-253.

71. Centers of Disease Control and Prevention - CDC. Infection Prevention during Blood Glucose Monitoring and Insulin Administration. I Injection Safety | CDC. [Internet]. [acesso em: 05 jan 2017] 2016. Disponível em:

https://www.cdc.gov/injectionsafety/blood-glucose-monitoring.html.

72. Brasil. Ministério do Trabalho e Emprego. Portaria no 485, de 11 de novembro de 2005. Aprova a norma regulamentadora no 32 (Segurança e saúde no trabalho em estabelecimentos de saúde) [Internet]. Diário Oficial da República Federativa do Brasil, Brasília(DF); 2005 Nov 11 [acesso: 2010 Ago 25]. Disponível em:

http://www.mte.gov.br/legislacao/normas regulamentadoras/nr 32.pdf.

73. Novaretti MCZ, Santos EV, Quitério LM, Daud-Gallotti RM. Sobrecarga de trabalho da Enfermagem e incidentes e eventos adversos em pacientes internados em UTI. Rev. bras. Enferm [online]. 2014. [acesso 07 mar 2017]; 67(5): 692-699. Disponível em: http://dx.doi.org/10.1590/0034-7167.2014670504. 